TRIBUNAL EUROPEO DE DERECHOS HUMANOS 



\title{
DE LA CUESTIÓN PREJUDICIAL DE CONVENCIONALIDAD EN MARCHA ${ }^{1}$
}

\author{
ANTONIO LÓPEZ CASTILLO \\ Catedrático de Derecho Constitucional \\ Universidad Autónoma de Madrid
}

JÖRG POLAKIEWICZ

Profesor de Derecho Público

Instituto Europeo de Sarrebruk

Director de Asesoría Jurídica y Derecho Internacional Publico

Consejo de Europa

\section{SUMARIO}

I. Introducción. II. Del Protocolo núm. 16 a la luz de sus antecedentes. III. Del primer dictamen prejudicial de convencionalidad. IV. Consideraciones finales, a modo de conclusiva recapitulación

\section{INTRODUCCIÓN}

El ex presidente del Tribunal Europeo de Derechos Humanos (TEDH), Dean Spielmann, al denominar al Protocolo 16 como «el protocolo del diálogo», acertó a caracterizarlo como esa vía de potencial articulación funcional entre el TEDH y los (supremos) tribunales nacionales que, en el espacio convencional europeo, sobre todo a partir de la Declaración de Brighton, tanto se había venido demandando.

Pues bien, mediante la entrada en vigor del referido Protocolo 16, previa apertura a la firma a principios de octubre de 2013, una vez ratificado, conforme

1 Comentario del Dictamen TEDH, Gran Sala, de 10 de abril de 2019 (demanda n. ${ }^{\circ}$ P16-2018-001), cuya versión española, se toma como referencia para cita. Las opiniones expresadas en este artículo solo responden al criterio de sus autores y no reflejan necesariamente la posición oficial del Consejo de Europa. Se trata de una contribución en el marco del Proyecto I+D sobre «El control de la ley» (DER 2016-78391-P). 
a lo dispuesto en su artículo 8, por los primeros diez Estados —Francia incluida ${ }^{2}$-, a partir del día 1 de agosto de 2018 quedaría ya expedita esa complementaria vía de comunicación procesal con el TEDH. Y, en pleno estío de 2019 —al cerrar estas páginas-, el estado de aceptación del protocolo núm. 16 bien puede decirse que se encuentra a medio camino. Pues, si bien es cierto que algo más de la mitad de los Estados (24, España entre ellos ${ }^{3}$ ) y la propia UE todavía no han procedido a su firma, los signatarios suman ya la cifra de 22 Estados, 13 de los cuales, según es notorio, habrían procedido ya a su ratificación.

En lo que sigue, previa referencia a los antecedentes del Protocolo (II), se tratará de la primera invocación (francesa) de este instrumento dialógico. Mediante el planteamiento de esa primera consulta prejudicial (medida, si no del todo concreta), el Tribunal de Casación, una de las tres supremas instancias (jurisdiccionales) franceses habilitadas ${ }^{4}$, plantearía dos cuestiones relativas al anclaje convencional de la relación paterno filial con la madre comitente de niño nacido en el extranjero, en un caso de acordada gestación subrogada y acerca de su régimen de inscripción registral en Francia.

Más concretamente, mediante decisión de 5 de octubre de 2018, la Corte de Casación pretendía averiguar, en primer lugar, «si el derecho al respeto de la vida privada, en el sentido del artículo 8 del Convenio, de un niño nacido en el extranjero a través de un acuerdo de gestación subrogada, que exige que la relación jurídica entre el niño y el padre comitente, cuando éste sea el padre biológico, sea reconocida en la legislación nacional, también exige que la legislación nacional ofrezca la posibilidad de reconocer la relación jurídica paterno filial con la madre comitente, que en el certificado de nacimiento legalmente establecido en el extranjero sea designada como «madre legítima», en una situación en la que el niño sea concebido utilizando los óvulos de una tercera donante y en la que la relación jurídica paterno-filial con el padre comitente fuese reconocida en la legislación nacional»; y, en segundo lugar (caso de responderse afirmativamente

2 A esas primeras ratificaciones (Albania, Armenia, Eslovenia, Estonia, Finlandia, Georgia, Lituania, San Marino, Ucrania y la ya referida de Francia), se vienen a sumar, ya en 2019, los casos de Países Bajos, Grecia y Andorra, con efectos a partir de 1 de junio, 1 de agosto y 1 de septiembre, respectivamente.

3 A propósito del debate abierto en España cf., como contribución más reciente, Matia Portilla, F.J., «¿Resulta oportuno dar un tratamiento jurídico a la gestación subrogada en nuestro país?», Revista de Derecho Político, n. ${ }^{\circ}$ 105, pp. 81-125. Y, asimismo, entre muchos otros, cf. Díaz Romero, M. ${ }^{a}$ R., Autonomía de la voluntad y contrato de gestión subrogada. Efectos jurídicos, Pamplona, Aranzadi, 2018; RuIz-Rico Ruiz, G., «La problemática constitucional derivada de las técnicas de reproducción humana asistida (TRHA): el caso de la maternidad subrogada», Revista de Derecho Político, n. ${ }^{\circ}$ 99, 2017, pp. 49-78; SALAZAR Benítez, O., «La gestación por sustitución desde una perspectiva jurídica: algunas reflexiones sobre el conflicto entre deseos y derechos», Revista de Derecho Político, n. ${ }^{\circ}$ 99, 2017, pp. 79-120; Vilar GonZÁlez, S., «Las sentencias de 27 de enero de 2015 y de 24 de enero de 2017 del Tribunal Europeo de Derechos Humanos en el caso 'Paradiso y Campanelli contra Italia' y la vulneración del derecho a la vida privada y familiar en materia de gestación subrogada», Revista de Derecho y Genoma Humano, n. ${ }^{\circ} 46,2017$, pp. 235-249.

4 Siendo los otros dos el Consejo constitucional y el Consejo de Estado, según se desprende de la Declaración que acompaña al instrumento de ratificación depositado con fecha 12 de abril de 2018. 
a la primera pregunta), «si el derecho del niño al respeto de su vida privada en el sentido del artículo 8 del Convenio exige que dicho reconocimiento adopte la forma de inscripción en el registro de nacimientos, matrimonios y defunciones de los datos del certificado de nacimiento legalmente constituido en el extranjero, o si se podría permitir que se utilizaran otros medios, como la adopción del niño por parte de la madre comitente» ${ }^{5}$.

Pues bien, en lo que sigue (III), tras un apunte preliminar (1.), se dará cuenta de la medida respuesta prejudicial brindada por el TEDH a propósito de la consulta relativa al muy controvertido asunto de la gestación por sustitución (2.). Y, acto seguido, a modo de conclusión (IV), sin perder de vista este primer dictamen prejudicial, se volverá sobre la caracterización y funcionalidad de la dialógica variante de comunicación convencional, inserta mediante el Protocolo 16, en este singular espacio europeo de confluencia entre Tribunales ${ }^{6}$; así que al ordinario modo de aplicación de los estándares convencionales sentados por el TEDH, mediante adopción, en su caso (en supuestos de falencia sistémica o recurrente vulneración de las cláusulas del CEDH), de medidas de alcance general, se suma ahora un extraordinario cauce consultivo de preventiva elusión de insuficiencias y desajustes de los estándares nacionales con respecto a unos estándares convencionales de referencia no siempre acomodados a la retícula europea de jurisdicciones en concurso ${ }^{7}$.

\section{DEL PROTOCOLO NÚM. 16 A LA LUZ DE SUS ANTECEDENTES}

Se recordará que la propuesta de ampliar la competencia del TEDH para emitir opiniones consultivas se formuló por primera vez en el informe al Comité de Ministros del Grupo de Sabios, creado en virtud del Plan de Acción adoptado en la Tercera Cumbre de Jefes de Estado y de Gobierno de los Estados miembros del Consejo de Europa (Varsovia, 16-17 de mayo de 2005) «para examinar la cuestión de la eficacia a largo plazo del mecanismo de control del CEDH.»

El Grupo de Sabios concluyó que «sería útil introducir un sistema en virtud del cual los tribunales nacionales pudieran solicitar al Tribunal opiniones consultivas sobre cuestiones jurídicas relativas a la interpretación de la Convención y sus protocolos, a fin de fomentar el diálogo entre los tribunales y reforzar la

5 Vid. Dictamen de la Gran Sala, de 10 de abril de 2019, parágrafos 32 y 33.

6 Cf., por otros, López Castillo, A., «La confluencia de Tribunales constitucionales, TEDH y TJUE», Anuario de la Facultad de Derecho de la UAM, n. ${ }^{\circ} 22,2018$, pp. 133-172.

7 Para una primera aproximación al Protocolo n. ${ }^{\circ} 16$ y a esta primera consulta prejudicial de convencionalidad cf., por ejemplo, el comentario de Durán Ayago, A., «Protocolo n. ${ }^{\circ} 16$ al Convenio Europeo de Derechos Humanos y Gestación por sustitución: primera consulta planteada por la Corte de Casación francesa y primera respuesta para seguir sin desbrozar del todo el camino. (RI \$421437)», Revista General de Derecho Europeo, n. ${ }^{\circ} 48,2019$. 
función 'constitucional' del Tribunal. Las solicitudes de opinión, que serían presentadas únicamente por los tribunales constitucionales o los tribunales de última instancia, serían siempre facultativas y las opiniones emitidas por el Tribunal no serían vinculantes.» ${ }^{8}$. La propuesta del Grupo de Sabios fue examinada por el Comité Directivo para los Derechos Humanos (CDDH) como parte de su trabajo de seguimiento del informe del primero. ${ }^{9}$

Posteriormente, la Conferencia de Alto Nivel de Esmirna sobre el futuro del TEDH (26-27 de abril de 2011), en su Declaración final, «[invitaría] al Comité de Ministros a reflexionar sobre la conveniencia de introducir un procedimiento que permita a los más altos tribunales nacionales solicitar opiniones consultivas de la Corte sobre la interpretación y aplicación de la Convención que ayuden a aclarar las disposiciones de la Convención y la jurisprudencia de la Corte», proporcionando, así, una orientación adicional al efecto de contribuir a evitar futuribles vulneraciones del CEDH por parte de los Estados signatarios ${ }^{10}$.

El informe final del CDDH al Comité de Ministros sobre las medidas que requieren la modificación del $\mathrm{CEDH}^{11}$ incluía un examen en profundidad de una propuesta más detallada presentada por los expertos de los Países Bajos y Noruega, reflejada también en su contribución a la Conferencia Ministerial organizada por la Presidencia del Comité de Ministros del Reino Unido. ${ }^{12}$

La cuestión de las opiniones consultivas se debatió ampliamente durante la preparación de la posterior Conferencia de Alto Nivel de Brighton sobre el futuro del Tribunal (19-20 de abril de 2012), a la que el TEDH contribuyó con un detallado 'Documento de reflexión sobre la propuesta de ampliar la competencia consultiva del Tribunal'. ${ }^{13}$

La Declaración final de la Conferencia de Brighton, «[tomando nota] de que la interacción entre el Tribunal y las autoridades nacionales podría reforzarse mediante la introducción en la Convención de un nuevo poder del Tribunal, que los Estados Partes podrían aceptar opcionalmente, para emitir dictámenes consultivos, previa solicitud, sobre la interpretación de la Convención en el contexto de un caso específico a nivel nacional, sin perjuicio del carácter no vinculante de los dictámenes de los demás Estados Partes[, invitó] al Comité de Ministros a redactar el texto de un protocolo facultativo de la Convención a tal efecto antes

8 Véase el doc. CM(2006)203, para. 135.

9 Véase el Informe de actividad del CDDH sobre la garantía de la eficacia a largo plazo del sistema de control del Convenio Europeo para la Protección de los Derechos Humanos, doc. CDDH(2009)007 Adición I, párrs. 42-44 y el dictamen del CDDH sobre los temas que se tratarán en la Conferencia de Interlaken, doc. CDDH(2009)019 Addendum I, párr. 1. 19.

10 Véase el doc. CM/Del/Dec(2011)1114/1.5. Estas instrucciones se incorporaron posteriormente en el mandato para el bienio 2012-2013 del órgano subordinado del CDDH, el Comité de expertos sobre la reforma de la Corte (DH-GDR).

11 Véase el doc. CDDH(2012)R74 Addendum I, párrs. 51-56 y Apéndice V.

12 Véase el doc. CDDH(2012)R74 Addendum III, para. 17.

13 Ver doc. \# 3853038, 20 de febrero de 2012. 
de finales de 2013; y a decidir, a continuación, si se aprueba o no». Tras la Conferencia de Brighton, el Comité de Ministros en su $122^{\circ}$ sesión ministerial (23 de mayo de 2012) encargaría al CDDH que redactara el texto necesario, lo que se hizo inicialmente mediante dos reuniones de un Grupo de Redacción de composición restringida, antes de su pase a examen del Comité de expertos sobre la reforma de la Corte (DH-GDR) en sesión plenaria, tras lo cual el proyecto fue examinado y aprobado por el $\mathrm{CDDH}$ en su $77^{\mathrm{a}}$ reunión (22 de marzo de 2013 ) para su presentación al Comité de Ministros.

La Asamblea Parlamentaria, por invitación del Comité de Ministros, aprobó la Opinión $N^{\circ} 285$ (2013) sobre el proyecto de protocolo el 28 de junio de 2013. En su $1176^{a}$ reunión, el Comité adoptaría ya el proyecto, como Protocolo $\mathrm{N}^{\circ} 16$ del Convenio (STCE N²14).

Entre las cuestiones abordadas durante todo ese proceso de negociación cabe destacar lo relativo a la naturaleza de la autoridad interna que pudiera solicitar una opinión consultiva del TEDH, así como al tipo de cuestiones sobre las que la Corte hubiera de emitir opinión consultiva; y, ya en otro plano, lo atinente al procedimiento para examinar las solicitudes, para deliberar sobre las solicitudes aceptadas y para emitir opiniones consultivas; y, como cuestión particularmente relevante, todo lo relativo al alcance de sus efectos jurídicos sobre las diferentes categorías de casos posteriores.

Y acaso deba considerarse esta última, la cuestión de los efectos jurídicos de los dictámenes del Tribunal, como la más enjundiosa de todo ese debate. Pues, sin perjuicio del temprano acuerdo acerca de que esos dictámenes prejudiciales no vinculasen a los tribunales solicitantes (lo que se explicitaría, finalmente, ex artículo 5 del Protocolo 16), se reconocería que esas interpretaciones prejudiciales de las disposiciones del Convenio por parte del TEDH vendrían a «formar parte de la jurisprudencia del Tribunal, junto con sus sentencias y decisiones»; o, dicho en otros términos, que la interpretación del Convenio y de sus Protocolos contenida en esos dictámenes se habría de considerar «análoga, por sus efectos, a los elementos interpretativos establecidos por el TEDH en sus sentencias y decisiones» ${ }^{14}$.

De la relevancia de los criterios de principio enunciados en esos dictámenes, a modo de registro puntual y concreto, en un determinado momento, tendrían el peso específico que resulta del hecho de su sistemático pronunciamiento por parte de la Gran Sala. Como parece haber dicho el experto británico durante las negociaciones, serían algo así como el «el salmón del sándwich de salmón».

Ello cobra sentido si se atiende a la evidencia de que el Convenio y sus Protocolos no son simplemente un catálogo de derechos y libertades fundamentales básicos, sino que constituyen un corpus jurídico que ha sido probado, desarrollado y aplicado por el Tribunal durante decenios. Así como, al hecho notorio de

14 Informe explicativo $§ 27$. 
que, en su jurisprudencia, el Tribunal aborda numerosos problemas críticos en materia de tutela de derechos humanos, ya se trate de la tortura y de los tratos inhumanos o degradantes, o del uso de armas de fuego por parte de la policía, ya se trate de las garantías de un juicio justo, ya se trate de la emergente inmigración, o de los derechos de los niños y de los padres, o de la privacidad de los datos personales, o de los derechos de los LGTBI, etc.

Por lo demás, se ha de recordar cómo al decidir sobre el fondo de un recurso, el Tribunal de Estrasburgo, de ordinario, trata de la naturaleza y el alcance de las obligaciones que se derivan de los derechos y libertades a examen. Y se ha de recordar, asimismo, que la fuerza vinculante de las sentencias (res judicata) es una cosa y que otra cosa habrá de ser su potencial valor como precedente para casos similares ${ }^{15}$. Esta finalidad de las sentencias del Tribunal habría sido ya explícitamente reconocida por los Estados cuando remitieron casos al Tribunal en virtud del artículo 48 de la Convención original. ${ }^{16} \mathrm{Y}$ el TEDH ha subrayado sistemáticamente que sus «sentencias no sólo sirven para resolver los asuntos sometidos al Tribunal, sino también, de manera más general, para dilucidar, salvaguardar y desarrollar las normas instituidas por el Convenio, contribuyendo así al cumplimiento por parte de los Estados de los compromisos que han asumido en su calidad de Estados contratantes.» ${ }^{17} \mathrm{Y}$, en fin, en otras sentencias, ha insistido el Tribunal en que su jurisprudencia previa habría aclarado la naturaleza y alcance de las obligaciones de las Partes en virtud del Convenio. ${ }^{18}$

15 A propósito de esto, el primer juez alemán del TEDH, Mosler, H. acuñó el término «Orientierungswirkung» en el V Coloquio Internacional sobre el Convenio Europeo de Derechos Humanos, en el que también intervino STEIN, T., en Fráncfort del Meno, 1980 (véase Europäischer Menschenrechtsschutz Schranken und Wirkungen (1982), 366; Ch. Grabenwarter Europäische Menschenrechtskonvention, ${ }^{4 a}$ edición, 2009, § 16, nota marginal 9; véase también REss, G., «Supranationaler Menschenrechtsschutz und der Wandel der Staatlichkeit», Zä̈RV, n. ${ }^{\circ}$ 64, 2004, pp. 621-639, que, a propósito, habla de un ,efecto cuasi erga-omnes'). Ahora bien, entre una simple orientación indicativa y la sanción por la negativa a acatar una sentencia definitiva del TEDH (cf., a propósito, la primera decisión ex artículo 46.4 del CEDH, adoptada con fecha 19 de mayo de 2019 por la que se declara un incumplimiento de Azerbaiyán), cabe considerar los efectos positivos o prejudiciales de la cosa juzgada de una decisión consultiva en la medida en que, sin cerrar la vía de acceso ex post ante el TEDH se delimita un campo posible de actuación por parte de los poderes públicos.

16 Véase, por ejemplo, el caso König, Serie B n²5, 162[Alemania]; el caso Winterwerp, Serie B n 2. 31, 54[Países Bajos]; caso Schiesser, Serie B no. 32, 10[Suiza]; Caso Le Compte et al 38, 65[Bélgica]; Caso Campbell y Cosans, Serie B no. 42, 61[Reino Unido]; Caso Droogenbroek, Serie B no. 44, 39[Bélgica]; caso Sporrong y Lönnroth, Serie B no. 46, 10[Suecia].

17 Sentencia de 18.1.1978, Serie A no. 25, § 154; Guzzardi v. Italia, 6 de noviembre de 1980, § 86, Serie A no. 39; Karner v. Austria, no. 40016/98, § 26, TEDH 2003IX-; Rantsev c Chipre y Rusia, 7 de enero de 2010, no. $25965 / 04 \$ 197$.

18 Véase, por ejemplo, Pentidis y otros contra Grecia, sentencia de 9.6.1997, Reports of Judgments and Decisions 1997-III, 990 ( $\$ 19$ ); asunto Moreira de Azevedo, sentencia de 23.10.1990, serie A, $\mathrm{n}^{\circ} 189, \mathrm{n}^{\circ} 73 ;$ B. contra Austria, sentencia de 28.3.1990, serie A, $\mathrm{n}^{\circ} 175, \mathrm{n}^{\circ}$ 54; asunto Martins Moreira, sentencia de 26.10.1988, Serie A no. 143, § 60; caso De Cubber, sentencia (artículo 50) de 14.9.1987, Serie A no. 124 - B, § 21; caso Lingens, sentencia de 8.7.1986, Serie A no. 103, § 46; caso Guincho, sentencia de 10.7.1984, Serie A no. 81, $\S 38$; caso Zimmermann y Steiner, sentencia de 13.7.1983, Serie A no. 66, § 32. 
Relativamente temprana es la opinión de que la interpretación de un determinado artículo, desarrollada por el TEDH en una serie de casos individuales, trasciende de los hechos particulares de estos casos, deviniendo parte integrante de dicha disposición y participando, en consecuencia, de la misma fuerza vinculante que la disposición examinada ${ }^{19}$. De modo que, en su desarrollo, esa evolutiva jurisprudencia del TEDH, lejos de considerarse una especie de fungible regla judicial $^{20}$, de ordinario, integra el sentido y alcance de las diversas cláusulas del CEDH. En fin, sin negar la proyección de esa comprensiva lectura a propósito de sus dictámenes prejudiciales, lo cierto es que ello se refleja mejor en el informe explicativo que en el texto (cf. artículo 5) del Protocolo núm. 16.

\section{DEL PRIMER DICTAMEN PREJUDICIAL DE CONVENCIONALIDAD}

\section{Apunte preliminar}

Previa aceptación, a cinco jueces, de la solicitud prejudicial, con fecha 3 de diciembre de 2018, el Presidente de la Gran Sala abriría el plazo para la presentación de observaciones escritas. A lo que respondieron los Mennesson ${ }^{21}$, el Gobierno francés y algunos otros Gobiernos y entidades varias. Conclusa esa fase escrita, sin intervención adicional alguna por parte del Tribunal de Casación, se excluiría la celebración de vista oral.

Al hilo de su aducida evolución jurisprudencial, atendiendo además al estado de la cuestión en el ámbito ius internacional, público y privado, en relación con el estatuto del menor y con el complejo asunto de que aquí se trata, el Tribunal de casación decidiría acudir al TEDH en busca de un estándar convencional seguro $^{22}$; al efecto de superar la relativa indefinición mennessoniana acerca del modo de integración de la identidad y de su instrumental cláusula de filiación, como elementos propios del derecho a la intimidad, con la imperiosa directriz del interés superior del menor ${ }^{23}$.

19 Véase L. Wildhaber ,Erfahrungen mit der Europäischen Menschenrechtskonvention ' Revue de droit suisse, 98 (1979) II, 329 (355); Ress, op. cit. supra nota 1, en The European System, 811-812; R. Bernhardt, The Convention and Domestic Law, en R. St-J. Macdonald/F. Matscher/H. Petzold (eds.) The European System for the Protection of Human Rights (Dordrecht, Nijhoff, 1993), 25 (39); P. Leuprecht 'The Execution of Judgments and Decisions' ibid., 791, at 792-793.

20 Valenzuela Contreras v. España, sentencia de 30.7.1998, Informes de sentencias y decisiones 1998-V, $1928 \S 60$ (escuchas telefónicas).

21 Cf., para un detalle más preciso, parágrafos 4-6 y 7-8 del Dictamen.

22 Cf., para un detalle más preciso, Dictamen TEDH, parágrafos 10-18 y 19-21.

23 Cf., en particular, parágrafos 96 y 99 Sentencia TEDH, Mennesson c. France, expresamente citados en parágrafo 12 del Dictamen TEDH. Y, a mayor abundamiento, en esa línea ha seguido pronunciándose el TEDH en sucesivas ocasiones (cf. las referencias a los casos Labassee c. Francia, de 26 de junio de 2014; 
En este caso, teniendo en cuenta que los procedimientos nacionales se refieren al reconocimiento en el ordenamiento jurídico francés de una relación jurídica paterno filial entre una madre comitente y un hijo nacido en el extranjero mediante un acuerdo de gestación subrogada, concebido utilizando los gametos del padre comitente y de una tercera donante, las cuestiones planteadas por el Tribunal de Casación ni siquiera se refieren a situaciones que impliquen acuerdos tradicionales típicos de gestación subrogada, esto es, aquellos compromisos en virtud de los que el niño fuese concebido utilizando los óvulos de la gestante subrogada ${ }^{24}$.

En este punto, si no se tratase — como se trata aquí- solo de presentar sumariamente los rasgos más destacables del primer dictamen prejudicial del TEDH, se haría preciso dar cuenta de la complejidad de un asunto que, a la dificultosa tarea de contraste comparatista, de la que el propio TEDH algo deja apuntado $^{25}$, suma la inexcusable exigencia de una mínima clarificación conceptual $^{26}$.

\section{Respuesta prejudicial}

A propósito del derecho a la vida privada que ex artículo $8 \mathrm{CEDH}$ se reconoce al niño nacido en el extranjero, mediante un acuerdo de gestación subrogada, y de su potencial exigencia de ampliación del reconocimiento de la relación de filiación, que la legislación nacional ya reconoce al padre comitente, a la «madre legítima» — - es decir, a la madre comitente así designada en el certificado de nacimiento legalmente establecido en el extranjero (en donde se concibiera el niño mediante el empleo de gametos del padre comitente y de una tercera persona donante) — el TEDH ha respondido de modo afirmativo, pero no sin modular el sentido y alcance de su respuesta.

Se reconoce, de inicio, que el derecho al respeto de la vida privada que convencionalmente se reconoce al niño comporta exigencias para el legislador nacional. Ahora bien, advirtiendo acerca de la constatable falta de consenso europeo (lo que se ilustra subrayando la diversidad de respuestas jurídicas en unos u otros países), expresamente se niega que del artículo $8 \mathrm{CEDH}$ pudiera resultar para Francia - en este caso- otra obligación convencional que la de exigencia de previsión de reconocimiento de una relación filial con la madre comitente (madre no

Foulon y Bouvet c. Francia, de 21 de junio de 2016; Laborie c. Francia, de 19 de enero de 2017, en parágrafo 35 del presente Dictamen TEDH).

24 Vide, para un detalle más preciso, parágrafos 27-29.

25 Cf., para un detalle más preciso, parágrafos 22-24.

26 A propósito, por su meritorio esfuerzo terminológico, bien puede remitirse aquí al extenso y bien documentado estudio «El régimen de subrogación en los Estados miembros de la UE», elaborado por BRUNET, L. et al., por encargo del PE, Dirección General de políticas interiores, Departamento temático C (Derechos de los ciudadanos y asuntos constitucionales), 2013, disponible en <http://www.europarl.europa.eu/ studies $>$. 
biológica que — se ha de recordar- en el documento que certificara el nacimiento subrogado literalmente se designa como «madre legítima»).

Pero, a fin de cohonestar esa exigencia convencional y la salvaguarda del margen de discrecionalidad estatal remanente — atendida la referida falta de consenso europeo en este campo-, esa obligación estatal de reconocimiento no se habría de traducir necesariamente en una exigencia de «inscripción en el registro de nacimientos (...) de los datos de la partida de nacimiento legalmente establecida en el extranjero»; pudiendo satisfacer el legislador esa obligación convencional por «otros medios, como la adopción del niño por la madre comitente, siempre que el procedimiento establecido por la legislación nacional garantice que pueda ser aplicado con prontitud y eficacia, de conformidad con el interés superior del niño» ${ }^{27}$.

En el Dictamen del TEDH se habría declarado, a propósito, que, como quiera que el derecho a la vida privada no lo sería todo (o, más precisamente, que el mismo sería solo una parte de la cuestión), la pretendida invocación del margen de discrecionalidad de los Estados parte en el CEDH no podría ser objeto de consideración al margen de «las circunstancias, el objeto y el contexto» de la cuestión examinada ${ }^{28}$. Lo que, en este caso ( «con más razón» que en otros), le lleva, primero, a dejar sentado lo que no cabe, y segundo, a precisar lo que sí cabe.

Pues, una vez afirmado que «la (hipotética) imposibilidad general y absoluta de obtener el reconocimiento de la relación entre un niño nacido por medio de un contrato de gestación subrogada en el extranjero y la madre comitente es incompatible con el interés superior del niño, que exige, como mínimo, que cada situación sea examinada a la vista de las circunstancias particulares del asunto» ${ }^{29}$, se precisa que la invocación del interés superior del niño «no puede interpretarse en el sentido de que el reconocimiento de la relación jurídica paterno-filial entre el niño y la madre comitente (ex artículo $8 \mathrm{CEDH}$ ), implique la obligación de que los Estados registren los detalles del certificado de nacimiento extranjero (pues dependiendo de las circunstancias de cada caso, otros medios también pueden servir a esos intereses de manera adecuada, incluida la adopción (de) efectos similares al del registro de (esos) datos.» ${ }^{30}$; eso sí, «siempre que las condiciones... sean adecuadas y el procedimiento permita adoptar rápidamente una decisión» ${ }^{31}$ pues «las alternativas al registro (y) en particular la adopción por parte de la madre comitente (solo) pueden considerarse aceptables en la medida en que el procedimiento establecido por la

\footnotetext{
27 Para un detalle más preciso se remite al enunciado final de la opinión emitida por el TEDH.

28 Para un detalle más preciso, cf. parágrafo 43, Dictamen TEHD.

29 Citados del parágrafo 42 (y 47).

30 Citado del parágrafo 53, Dictamen TEDH.

31 Citado del parágrafo 54, Dictamen TEDH.
} 
legislación nacional garantice (su) prontitud y eficacia, de conformidad con el interés superior del niño» ${ }^{32}$.

Dicho esto, la Gran Sala recuerda que — como es obvio— «en el marco de su Dictamen no le corresponde al Tribunal pronunciarse sobre si la normativa de adopción cumple (o no) los criterios enunciados en los (ya extractados) párrafos 54 y 55», que «esa es una cuestión que deben decidir los tribunales nacionales... teniendo en cuenta la posición vulnerable de los niños en cuestión mientras los procedimientos de adopción se encuentren pendientes $»^{33}$.

Dejando de lado «la complejidad de las cuestiones planteadas por los acuerdos de gestación subrogada» (algo de lo que — como el propio TEDH reconocese viene ocupando la Conferencia de la Haya sobre Derecho internacional privado) y partiendo de la estructural divisoria de jurisdicción en que se sustenta el sistema convencional de tutela de derechos y libertades fundamentales, se apunta en Gran Sala a las insuficiencias de un régimen de adopción que, sin perjuicio de su acreditada práctica ${ }^{34}$, comportaría insuficiencias, dada su restricta disponibilidad (excluyendo padres comitentes no casados), y dudas, por lo que a la manifestación de voluntad de la «madre de alquiler» se refiere ${ }^{35}$.

Acaso como respuesta (in)mediata a esta indirecta llamada de atención de la Gran Sala, ante un estado de la cuestión que, por definición, no es asunto de su competencia, se ha de considerar aquí la decisión de 23 de mayo de 2019, del Tribunal de Gran Instancia de Nantes, por la que se acuerda la plena «retranscripción» del estado civil de una niña nacida en los Estados Unidos de Norteamérica (EE.UU.) de padre con aquella ciudadanía y madre (de intención) de nacionalidad francesa.

Mediante esa decisión se trasciende ya del mínimo convencional inexcusable, declarado en el Dictamen prejudicial del TEDH, avanzando así, aun a falta de regulación legal, en la satisfacción del supremo interés del menor por la vía menos segura (estando esa decisión pendiente de apelación), mediante expreso reconocimiento de una situación constituida en el extranjero, de conformidad con aquella ley, como el modo más indicado de garantizar en el foro el derecho al respeto de la identidad de una menor, de la que su filiación y nacionalidad francesa constituirían elemento esencial ${ }^{36}$.

32 Citado del parágrafo 55, Dictamen TEDH.

33 Citado del parágrafo 58, Dictamen TEDH.

34 En el parágrafo 57, Dictamen TEDH, se recuerda según el alegato del gobierno galo «entre el 5 de julio de 2017 y el 2 de mayo de 2018, prácticamente todas las solicitudes de adopción del hijo del cónyuge relativas a hijos nacidos de madre de alquiler en el extranjero fueron aceptadas».

35 En el parágrafo 57, Dictamen TEDH, se ponen de manifiesto las matizaciones que, a propósito de su régimen de aplicación, expusieran el propio Tribunal de Casación y el Defensor del Pueblo francés.

36 Vide, a propósito, la somera referencia de Caroline Le Bot, Gestation pour autrui, enfin una reconnaissance de la «mère d`intention»?, accesible en <https:/www.village-justice.com/articles/gestation-pour-autrui-enfin-une-reconnaissance-mere-intention, 31629.html>. 


\section{CONSIDERACIONES FINALES, A MODO DE CONCLUSIVA RECAPITULACIÓN}

De las últimas conferencias intergubernamentales sobre el futuro del TEDH (Esmirna 2011, Brighton 2012, Bruselas 2015 y Copenhague 2018) surgió un mensaje claro en el que se invitaba al Tribunal a mostrar autocontrol sobre las cuestiones que mejor podían ser tratadas por los tribunales y cortes nacionales y las legislaturas elegidas democráticamente en los Estados miembros. Bien puede decirse, a propósito, que el TEDH haría bien en resistir «la tentación de la telaraña», renunciando a la pretendida construcción, con base en el $\mathrm{CEDH}$, de un sistema jurídico completo en el que los ordenamientos nacionales pudieran sentirse «atrapados» o «acorralados» ${ }^{37}$.

Conviene recordar, en este punto, que el CEDH siendo como es un tratado internacional que prevé la protección efectiva de un determinado número de derechos — ciertamente ampliado, en un doble sentido, cuantitativo y cualitativo, en virtud de sucesivos Protocolos y mediante la evolutiva jurisprudencia del TEDH-, no puede tenerse por un ordenamiento jurídico en el sentido en que pueda serlo el complejo normativo que se anuda a los Tratados de la Unión Europea ${ }^{38}$.

En el presente caso, los criterios enunciados por la Gran Sala, en particular, en sus parágrafos $54 .^{\circ}$ y $55 .^{\circ}$, ponen este novedoso instrumento procesal al servicio de la constitutiva divisoria funcional en el espacio de confluencia convencional. Pues, partiendo de esas indicaciones, se afirma expresamente que la resolución del caso concreto es asunto propio de la competencia, no del TEDH, sino de los Tribunales nacionales (pues, el objetivo del procedimiento no es la traslación del litigio interno al Tribunal, sino la búsqueda de orientación por parte del órgano jurisdiccional solicitante acerca de cuestiones relativas al Convenio requeridas de clarificación al efecto de resolver un concreto asunto ${ }^{39}$ ). Se reafirma, así, esa dupla de rasgos basilares del sistema convencional, que expresamente jalonan el Dictamen, la subsidiariedad de la jurisdicción del TEDH y el margen estatal de apreciación o remanencia de un cierto grado de discrecionalidad ${ }^{40}$.

37 Cf. P. Cruz Villalón, cit., 9-10.

38 A propósito del DUE como ordenamiento y de sus rasgos definitorios y caracteres (concebidos en la perspectiva del «como si»), me permito remitir a López CASTILlo, A. (dir.), Instituciones y Derecho de la Unión

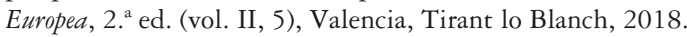

39 Una orientación de sólito confluente (y así ha sido en este primer caso), pero que bien podría llegar a formularse como expresión mayoritaria si es que, mediante controversia, llegase a formularse opinión discrepante (tal y como, con frecuencia, ocurre en el desempeño ordinario de su función jurisdiccional). Al órgano jurisdiccional solicitante corresponderá, por su parte, resolver las cuestiones planteadas por el asunto y extraer, en su caso, las conclusiones que se desprendan del dictamen emitido por el Tribunal sobre las disposiciones de Derecho nacional invocadas en el asunto y sobre el resultado del mismo.

40 Cf., con carácter general, por otras muchas, la sintética contribución de PAulus, A., en Conference on the long-term future of the European Cour of Human Rights, Proceedings/Actes, Oslo, 7-8 April 2014, pp. 55, 59-60. 
Y sentados los criterios paramétricos, mediante la especial consideración de la vulnerabilidad de la posición de los niños, a las autoridades nacionales se les deja la tarea de asegurar su acomodo, sin perjuicio de su margen nacional de apreciación al establecer disposiciones generales. Y a ellas mismas compete el efectivo control de convencionalidad de la ley mediante una praxis jurisprudencial, en la que se asegure el reconocimiento y efectiva aplicación de los mínimos estándares convencionales o alumbrando, en su caso, pluses de tutela. Pues, nada impide que los jueces nacionales vayan más lejos que el juez europeo ${ }^{41}$; y lo que decidan los tribunales nacionales, con no ser vinculante para Estrasburgo ni para ningún otro tribunal estatal, bien puede enriquecer la jurisprudencia, particularmente, en relación con asuntos abiertos al debate; cual es aquí el caso, ante la constatada falta de un consenso que, de abajo arriba, bien puede contribuir a gestar una consistente jurisprudencia estatal de la que el TEDH pueda extraer referentes al efecto de desarrollar su propia jurisprudencia ${ }^{42}$. Claro que del ordinario desempeño de la función jurisdiccional estatal no se ha de esperar una mimética aplicación de estándares convencionales ${ }^{43}$ en abierta disonancia con manifestaciones regulatorias de consensos políticos anclados en procesos democráticos (así, por ejemplo, a propósito de la decisión de convertirse en padres, y no solo — como ha sido aquí el caso- mediante un contrato de subrogación) ${ }^{44}$.

Como es bien notorio, el CEDH quiso sentar una «visión compartida» común de la legislación europea en materia de derechos humanos, concebido como el marco «dentro del cual los Estados pueden optar por diferentes equilibrios fundamentales entre el gobierno y los individuos ${ }^{45}$. Y, al desarrollar sus previsiones, el TEDH se asegura niveles de aceptación tanto más consistentes cuanto mayor respeto muestra en sus resoluciones por esa diversidad nacional de la que es cifra la cláusula estructural del margen de apreciación ${ }^{46}$.

41 Contrariamente a ese enfoque cauteloso («nada menos, pero ciertamente nada más») que defendiera LORD Brown en A - Skeini.

42 A propósito del uso del «consenso» por parte del TEDH, cf., entre otros muchos, Murray, J., "Consensus: Concordance or Hegemony of the Majority», en Dialogue between Judges, European Court of Human Rights, Strasbourg, Council of Europe, 2008, pp. 35-70; Wildhaber, L., Hjartarson, A. y Donnelly, S., "No consensus about consensus? The Practice of the European Court of Human Rights», Human Rights Law Journal, n. ${ }^{\circ} 33,2013$, pp. 248-263.

43 A propósito, entre otros muchos ejemplos, cabría referir aquí el caso británico de la salvaguarda de inmunidad de funcionarios ante tribunales civiles (STEDH, de 14 de enero de 2014, n. ${ }^{\circ} 34356 / 06$ y 34356/06, as. Jones/United Kingdom).

44 Cf. STEDH, de 10 de abril de 2007, as. Evans/UK, § 73: «El dilema central del presente caso es que se trata de un conflicto entre los derechos del artículo 8 de dos particulares: el solicitante y J. Además, los intereses de cada persona son totalmente incompatibles con los de la otra, pues si se permite al solicitante utilizar los embriones, J se verá obligado a convertirse en padre, mientras que si se mantiene la negativa o la retirada del consentimiento de $\mathrm{J}$, al solicitante se le negará la oportunidad de convertirse en padre genético. En las difíciles circunstancias de este caso, cualquier solución que adopten las autoridades nacionales frustraría totalmente los intereses de una u otra de las partes en el tratamiento de FIV».

45 Weiler cit., 107, en línea con el Preámbulo del CEDH.

46 Como es notorio, el empeño por la pretendida configuración de estándares mejores o más elevados suma a las dificultades conceptuales (cf., por otros, Dworkin, R., Taking Rights Seriously, 270, 
Precisamente por eso, en su papel de guardián de este común convencional en materia de derechos humanos, el TEDH está especialmente llamado a dictar resoluciones — Sir Nicolas Bratza dixit_ en aquellos «casos que revelan graves problemas de cumplimiento de la Convención a nivel nacional, casos que hacen avanzar nuestra comprensión de la legislación sobre derechos humanos, casos en los que está en juego la dignidad y/o la integridad física de las personas, casos que miden la salud del Estado de Derecho y la democracia en nuestras sociedades». Sin incurrir, por el contrario, en una deriva de ilimitada deferencia judicial, que dejase a las autoridades nacionales sin una orientación clara en cuanto a la aplicación de los intereses concurrentes en virtud del Convenio.

Del TEDH, como tribunal especializado en la salvaguarda de los derechos humanos se ha de esperar siempre un razonamiento fundado al efecto de orientar a las autoridades nacionales acerca de los principios generales que rigen la ponderación de los derechos e intereses en relación con un determinado conflicto. Un razonamiento que, cuanto más notorio parezca el sustrato moral o ético subyacente a temas abiertos al debate y acerca de los cuales no acabe de formarse un consenso europeo, resultaría inexcusable si se ha de observar, sobre la divisoria jurisdiccional ${ }^{47}$, esa constitutiva exigencia de corrección funcional que obsta la arrogación de atribuciones legislativas en vía jurisdiccional ${ }^{48}$. Por lo demás, la presunción de que un órgano legislativo no tuviera la intención de adoptar una regulación al efecto de contrariar las obligaciones que le incumben en virtud del Convenio, operativa en el caso de disposiciones legislativas de ambiguo tenor, resultaría ya contradictoria con la estructural exigencia de sujeción del juez a la ley, ínsita en la cláusula de Estado de Derecho, si de inaplicar

Harvard, Harvard University Press, 1977), tribulaciones en la práctica cuando los ensayos de ponderación se enredan en contraposiciones apenas universalizables, a propósito de libertad de expresión y privacidad e intimidad (cf. SSTEDH, de 24 de junio de 2004 y 7 de febrero de 2012, as. Caroline von Hannover c. Alemania, 1 y Caroline von Hannover c. Alemania, 2); STEDH de 16 de enero de 2014, $\mathrm{n}^{\circ}$ 13258/09, Uiio-Stenberg y S E otros $c$. Noruega, donde el Tribunal adoptó una sentencia disidente del Tribunal Supremo noruego en dos o tres ocasiones, señalando en particular que «aunque las opiniones pueden diferir sobre el resultado de una sentencia, cuando el ejercicio de ponderación haya sido realizado por las autoridades nacionales de conformidad con los criterios establecidos en la jurisprudencia del Tribunal, el Tribunal necesitaría razones de peso para sustituir su opinión por la de los tribunales nacionales»), o bien acerca de los derechos de propietarios e inquilinos (así, por ejemplo, LADY HALE en la conferencia Warwick Law Lecture 2013 ¿Cuál es el sentido de los derechos humanos? (28 de noviembre de 2013), disponible en http://supremecourt.uk/docs/speech-131128.pdf; cf. Manchester City Council vPinnock[2010] UKSC 45,[2010] 2 AC 104), etc.

47 Cf., entre otros posibles ejemplos, SSTEDH, asuntos Stubing/Alemania, n. ${ }^{\circ} 43547 / 08$, n. $^{\circ} 16072 / 06$ y 27809/08, de 12 de abril de 2012; Frienland Countryside Alliance e.a./United Kingdom, de 24 de noviembre de 2009; Handyside/Reino Unido, n. ${ }^{\circ}$ 5493/72, de 7 de diciembre de 1976.

48 En ese sentido, puede entenderse también la cautelosa aproximación en Gran Sala a propósito de la prohibición de la simulación del rostro en el espacio público, mediante ley, declarada constitucionalmente conforme, en la STEDH de 1 de julio de 2014, SAS/Francia (cf., en esa misma línea, con referencias adicionales, López Castillo, A., «De lo subjetivo a lo objetivo en la jurisprudencia convencional en dos ejemplos», Teoría y Realidad Constitucional, n. ${ }^{\circ}$ 42, 2018, pp. 513, 521-5). 
una disposición legislativa manifiestamente contraria a los estándares convencionales se tratase.

Pues bien, precisamente, a propósito de supuestos de presumible disonancia, si no manifiesta contradicción, con los estándares convencionales, puede resultar de utilidad el empleo de esta preventiva vía de comunicación procesal que es la cuestión prejudicial, a resultas de la cual bien podría procederse a integrar el parámetro convencional, de un modo seguro, al proceder a sentar una doctrina legal o a declarar el contenido y alcance constitucional de un equivalente parámetro ius fundamental.

Así, pensando en el sistema de controles normativos propio del ordenamiento nacional francés, aquí concernido, el Tribunal de Casación puede avanzar en la decantación de su doctrina legal de modo no disconforme con un estándar convencional que, en esta ocasión, siguiendo ese cauteloso modo que el TEDH adopta de ordinario a falta de consenso en una determinada materia, si bien no puede afirmarse que sea maleable, resulta suficientemente flexible.

Claro está que esa preventiva vía de acomodación de estándares convencionales y nacionales (legales o/y constitucionales) puede encontrar un límite insuperable en aquellos hipotéticos supuestos en los que, pareciendo estar indicada una cuestión prejudicial, los supremos intérpretes llamados a plantearla decidan no dar ese paso. Tal podría considerarse el caso, a propósito de la ley francesa de prohibición de simulación del rostro en el espacio público, en el tracto de cuya aplicación (como la de otras disposiciones de semejante tenor y alcance), bien podría llegarse a plantear una cuestión prioritaria de inconstitucionalidad que pudiera llevar al Consejo constitucional a replantear la cuestión ante el TEDH por esta mediata vía de la cuestión prejudicial de convencionalidad (pero para que eso se llegara a replantear, sería preciso volver sobre el sentido de una regulación democrática de carácter prohibitivo, antes que limitativo, y de una lectura idiosincrática de la cláusula (constitucional y convencional) de orden público.

En todo caso, que la invocación del estándar convencional, pretendidamente contravenido como consecuencia de su aplicación, pueda llevarse, mediante la activación ordinaria de su jurisdicción, si bien abre una mediata vía de casuístico control ex post de esa concreta regulación prohibitiva, no deja de plantear algunas dificultades. La primera, compartida con la preventiva prejudicial, es que la misma no es una vía de control de convencionalidad de la ley que, sin perjuicio de las consecuencias de su desatención, conforme a la divisoria constitutiva del sistema convencional, como tal, solo compete a la jurisdicción nacional concernida.

Quiere ello decir que, mediante la intervención autorizada del supremo intérprete constitucional (en el caso francés, el Consejo constitucional, directa o indirectamente, mediante la llamada cuestión prioritaria de inconstitucionalidad), una ley semejante bien podría declararse conforme a la Constitución nacional.

Y entonces, el TEDH, ex ante, en vía prejudicial - como ex post, en vía ordinaria de casuística tutela - se vería expuesto a la alternativa de declarar esa concreta 
regulación nacional contraria a los estándares del CEDH o, en otro caso (sobre idénticos presupuestos a los considerados aquí), entender que ante la falta de consenso en la materia, en atención a la salvaguarda de las exigencias democráticas en su procedimiento de aprobación, ese restrictivo ejercicio regulatorio no podría entenderse ajeno a los valores y principios que delimitan y limitan el efectivo ejercicio de los derechos reconocidos en el $\mathrm{CEDH}$.

De modo que, así como se espera de la jurisdicción nacional un desempeño de su función en conformidad con las cláusulas del CEDH, así también, de la jurisdicción de tutela del TEDH se demanda un esfuerzo por acomodar el sentido y alcance de las cláusulas del CEDH a las exigencias derivadas de los derechos fundamentales en su interrelación y en su relación con aquellos valores y principios del Estado constitucional en que los mismos se asientan y desarrollan.

Se precisaría, al efecto, de una efectiva labor de hermenéutica conciliación de los diversos elementos integrantes de las previsiones convencionales, del objeto y manifestaciones, pero también de las limitaciones, de los derechos reconocidos; mediante una tarea de concordancia práctica ${ }^{49}$ que, lejos de limitarse al encaje de las manifestaciones subjetivas de ius fundamentalidad encontradas, integrase los elementos del orden público convencional que, sobre los derechos y libertades, encarnan en esos valores y principios democráticos de los que se nutre el Estado constitucional de Derecho.

Así las cosas, se trate de una u otra opción alternativa, el resultado tendería a una misma solución, a la interpretación de la ley nacional de conformidad con las exigencias derivadas del compromiso convencional. Se alcance como consecuencia de una integración jurisdiccional estatal (inmediata o mediata, mediante intervención del TEDH, ex ante o ex post) del parámetro constitucional mediante el estándar convencional o se logre al hilo de la integración del parámetro convencional, si no de conformidad, en consonancia con uno u otro estándar constitucional.

Por ello, solo en la hipótesis de desatento desenvolvimiento del margen de apreciación, mediante ley o en vía jurisdiccional, se precisa la intervención del TEDH, como resorte de salvaguarda de esos estándares obligatorios. Y esto que, hasta ahora, solo se podía llegar a producir con ocasión de un inmediato examen convencional de concretos supuestos de aplicación, desde ahora, se podrá producir ya también mediante la preventiva vía que habilita la cuestión de prejudicialidad de convencionalidad.

En todo caso, dada lo discrecional de su eventual planteamiento y lo restricto de su presumible concepción (si es que su lectura se hace a la luz del artículo 43, segundo apartado, $\mathrm{CEDH}$, subrayando la gravedad de las cuestiones interpretativas

49 Cf., a propósito de su caracterización como criterio hermenéutico constitucional, HESSE, K., Fundaments of the Constitutional Law of the Federal Repubic of Germany, Müller, Heidelberg, 1999, nota marginal 72. 


\section{o aplicativas en cuestión ${ }^{50}$ ), pareciera aconsejable seguir de cerca su potencial reco- rrido convencional antes de extraer conclusiones apresuradas ${ }^{51}$.}

$$
* * *
$$

\section{TITLE: Preliminary ruling under the Convention}

ABstRACT: On the basis of its antecedents, the first preliminary ruling under the Convention issued by the ECtHR at the request of the French Court of Cassation is examined here in relation to the controversial issue of surrogacy and the resulting system of filiation, in this case with the mother principal. After a brief approximation to the answer given to the questions expressly raised, it is a question of recapitulating the meaning and scope of this novel way of preventive clarification of the conventional standard, without contradicting the presuposed requirements in the conventional system of protection of fundamental rights and freedoms by the instrumental principles of subsidiarity of the jurisdiction of the ECtHR and the national margin of appreciation.

Resumen: Se examina aqui, a partir de sus antecedentes, el primer dictamen prejudicial de convencionalidad dictado por el TEDH a instancia del Tribunal de casación de Francia, en relación con el controvertido asunto de la gestación subrogada y del régimen de filiación resultante, en este caso, con la madre comitente. Tras una somera aproximación a la respuesta brindada a las cuestiones expresamente planteadas, se trata de recapitular acerca del significado y alcance de esta novedosa vía de preventiva clarificación del estándar convencional, sin contrariar las exigencias presupuestas en el sistema convencional de tutela de derechos y libertades fundamentales por los instrumentales principios de la subsidiariedad de la jurisdicción del TEDH y del margen nacional de apreciación.

KEY WORDS: Protocol n. ${ }^{\circ} 16$, preliminary Question of conventionality, Surrogacy by agreement, Right to privacy of the child, Regime for the registration of the child's subsidiary relationship with the mother principal, First preliminary ruling from the ECtHR.

Palabras Clave: Protocolo n. ${ }^{\circ}$ 16, Cuestión prejudicial de convencionalidad, Gestación subrogada mediante acuerdo, Derecho a la intimidad del niño, Régimen de inscripción registral de su relación filial con la madre comitente, Primer dictamen prejudicial del TEDH.

FECHA DE RECEPCIÓN: 09.08.2019

FECHA DE ACEPTACIÓN: 11.09.2019

50 Para mayor precisión, cf. Informe explicativo del Protocolo núm. 16. Y en esa línea —se habría planteado_-, precisamente a propósito de esta cuestión prejudicial, LE MAIGAT, P. «Mère d’intention et gestation pour autrui: La Cour de cassation à l'épreuve du dialogue des juges et de la filiation désincarnée», en Gazzette du Palais, 6 novembre 2018 (accesible desde: <https://etudiant.lextenso.fr>), que solo ante problemas de alcance estructural o sistémicos, o en ausencia de todo precedente o en pos de cuestionar cierta deriva jurisprudencial del TEDH, debiera pensarse en el planteamiento de tan singular vía procesal de preventiva decantación de estándares convencionales.

51 A propósito, resultará de interés la resolución que el TEDH acuerde dar a la segunda consulta, planteada ya con fecha 9 de agosto de 2019, por el Tribunal Constitucional de Armenia, en relación con la constitucionalidad del artículo 300.1 del Código Penal armenio, relativo a la usurpación del poder del Estado al derrocamiento del orden constitucional o a la violación de la integridad territorial. 